\title{
USO DE OBSERVADORES DE ESTADO USANDO FILTRO DE KALMAN ESTENDIDO PARA INFERÊNCIA E CONTROLE DE TEMPERATURA EM UM TANQUE COM SISTEMA DE AQUECIMENTO
}

\author{
W. Y. A. BRANDÃO ${ }^{1}$, M. S. PEDRO ${ }^{1}$, P. R. A. MARIZ1 ${ }^{1}$ L. O. A. ROJAS, A. A. MORAIS \\ JÚNIOR $^{1}$ \\ ${ }^{1}$ Universidade Federal da Paraíba, Departamento de Engenharia Química \\ E-mail para contato: walteryanko@gmail.com
}

\begin{abstract}
RESUMO - Neste trabalho foi desenvolvida uma estratégia para construção de um sensor virtual (soft sensor) para estimativa, eleminação de sinais ruidosos e controle de temperatura de um processo de agitação e com sistema de aquecimento. Na construção do sensor virtual foi empregada a modelagem semiempírica, através do Filtro de Kalman Estendido (FKE). O modelo dinâmico de inferência utilizando FKE foi construído em ambiente Matlab-Simulink®. Foram ara implementados um controle convencional realimentação e um controle cascata de temperatura. Verificou-se que, mesmo com sinais ruidosos na entrada do processo, o FKE apresentou valores satisfatórios quanto aos parâmetros da Média Quadrática do Erro (MSE) e Raiz da Média Quadrática do Erro (RMSE). Por fim, analisou-se que o controle realimentação e cascata proporcionaram uma minimização da resposta transiente de temperatura; de fato, o controle cascata mostrou-se mias robusto por apresentar menor sobre-elevação percentual e menores valores de critérios de erros integrais.
\end{abstract}

\section{INTRODUÇÃO}

O controle automático de processo é fundamental no melhoramento e manutenção de produtos. O controle automático trata, dentre outros parâmetros, da manutenção das variáveis, das temperaturas, das pressões, dos fluxos, e das composições de processo em um valor desejado (SMITH e CORRIPIO, 2008). Devido à necessidade em manter variáveis em um valor requerido e também da dificuldade em realizar medições dessas variáveis, surgem então os sensores virtuais (soft sensors), que são modelos matemáticos implementados em softwares que utilizam medições secundárias para estimar variáveis de difícil medição. Na construção dos sensores virtuais, podem ser utilizados modelos de primeiro princípio (fenomenológicos), modelos de identificação e modelagem caixa cinza. Quando são utilizados modelos do tipo espaços de estados, é dado o nome de observador ou estimador de estados. O observador pode ser exato quando fornece o estado diretamente, ou assintótico quando seu limite tende ao estado exato para tempo infinito (MORAIS JÚNIOR, 2011).

Normalmente ocorre, devido a desgastes ou falhas nos sensores, ou mesmo interferência de sinais ou limitações físicas, diferenças entre os modelos simulados e dados obtidos diretamente de processos reais operando, caracterizando os ruídos (AGUIRRE, 2007). 
A medição ruidosa de uma variável de processo reflete os ruídos para o controlador, produzindo efeitos indesejáveis no elemento final de controle e podendo tornar inviável a implementação do controle. Para eliminar ou reduzir o efeito dos ruídos são adicionados filtros ao processo, tal como o Filtro de Kalman. Neste trabalho, foi desenvolvida uma metodologia para desenvolver um observador de estados utilizando o Filtro de Kalman Estendido (FKE) para estimar o valor da temperatura em um processo a partir de medições secundárias, com adição de ruído e filtragem de sinais. A metodologia desenvolvida foi empregada em um tanque de agitação com sistema de aquecimento, do qual foi feita a modelagem e simulação utilizando o software Matlab-Simulink®. Em seguida, foram testadas duas estratégias de controle em comparação com a condição de malha aberta.

\section{MODELAGEM E SIMULAÇÃO DO PROCESSO}

A modelagem do processo é definida por um conjunto de duas equações EDOs, balanços de energia no tanque e na serpentina de aquecimento. As equações do modelo foram discretizadas utilizando a aproximação por Euler, e são apresentadas na Equação 1 e 2 . Foi assumido que não há perdas de calor do sistema à vizinhança, o agitador é de mistura perfeita, possui volume e propriedades físicas constantes.

$$
\begin{aligned}
& T(k+1)=T(k)+\frac{h}{V \rho c_{v}}\left\{f(k) \rho c_{P} T_{i}+U A\left[T_{s}(k)-T(k)\right]-f(k) \rho c_{P} T(k)\right\} \\
& T_{s}(k+1)=T_{s}(k)+\frac{h}{C_{M}}\left\{w(t) \lambda-U A\left[T_{s}(t)-T(t)\right]\right\}
\end{aligned}
$$

A figura 1 mostra o processo, sendo os valores dos parâmetros e condições iniciais de operação do processo apresentados na Tabela 1 (SMITH e CORRIPIO, 2008).

Figura 1 - Tanque de Aquecimento. Fonte: Smith e Corrípio (2008).

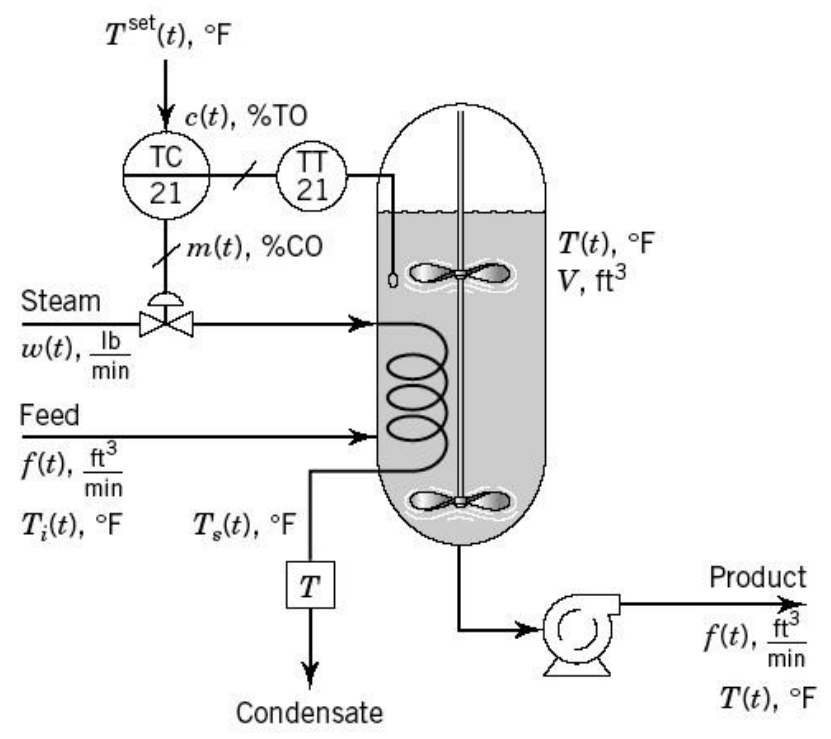




\section{Congresso Brasileiro de Engenharia Química \\ em Iniciação Científica \\ UFSCar - São Carlos - SP \\ 16 a 19 de Julho de 2017}

Tabela 1 - Parâmetros e condições operacionais do tanque de aquecimento.

\begin{tabular}{|c|c|c|c|c|c|}
\hline $\begin{array}{c}\text { Parâmetros e } \\
\text { condições } \\
\text { operacionais }\end{array}$ & $\begin{array}{l}\text { Valores e } \\
\text { unidades }\end{array}$ & $\begin{array}{c}\text { Parâmetros e } \\
\text { condições } \\
\text { operacionais }\end{array}$ & $\begin{array}{l}\text { Valores e } \\
\text { unidades }\end{array}$ & $\begin{array}{l}\text { Parâmetros e } \\
\text { condições } \\
\text { operacionais }\end{array}$ & $\begin{array}{l}\text { Valores e } \\
\text { unidades }\end{array}$ \\
\hline$\rho$ & $68 \mathrm{lb} / \mathrm{ft}^{3}$ & $\lambda$ & $\begin{array}{c}2,1 \\
\text { BTU/min. } \mathrm{ft}^{2}{ }^{\circ}{ }^{\circ} \mathrm{F}\end{array}$ & $\mathrm{T}_{\mathrm{S}}(0)$ & $230^{\circ} \mathrm{F}$ \\
\hline$c_{p}, c_{v}$ & 0,8 BTU/lb. ${ }^{\circ} \mathrm{F}$ & $\mathrm{C}_{\mathrm{M}}$ & $265,7 \mathrm{BTU} /{ }^{\circ} \mathrm{F}$ & \multirow{3}{*}{$\mathrm{T}(0)$} & \multirow{3}{*}{$150^{\circ} \mathrm{F}$} \\
\hline $\mathrm{V}$ & $120 \mathrm{ft}^{3}$ & $f(0)$ & $15 \mathrm{ft}^{3} / \mathrm{min}$ & & \\
\hline $\mathrm{A}$ & $241,5 \mathrm{ft}^{2}$ & $\mathrm{w}(0)$ & $42,2 \mathrm{lb} / \mathrm{min}$ & & \\
\hline
\end{tabular}

O fluxograma da Figura 2 apresenta a proposta de estimativa, eleminação de sinais de ruidosos na entrada, vazão de alimentação, e implmentação de controle inferencial cascata de temperatura.

Figura 2 - Fluxograma de simulação do processo e estimativa por FKE.

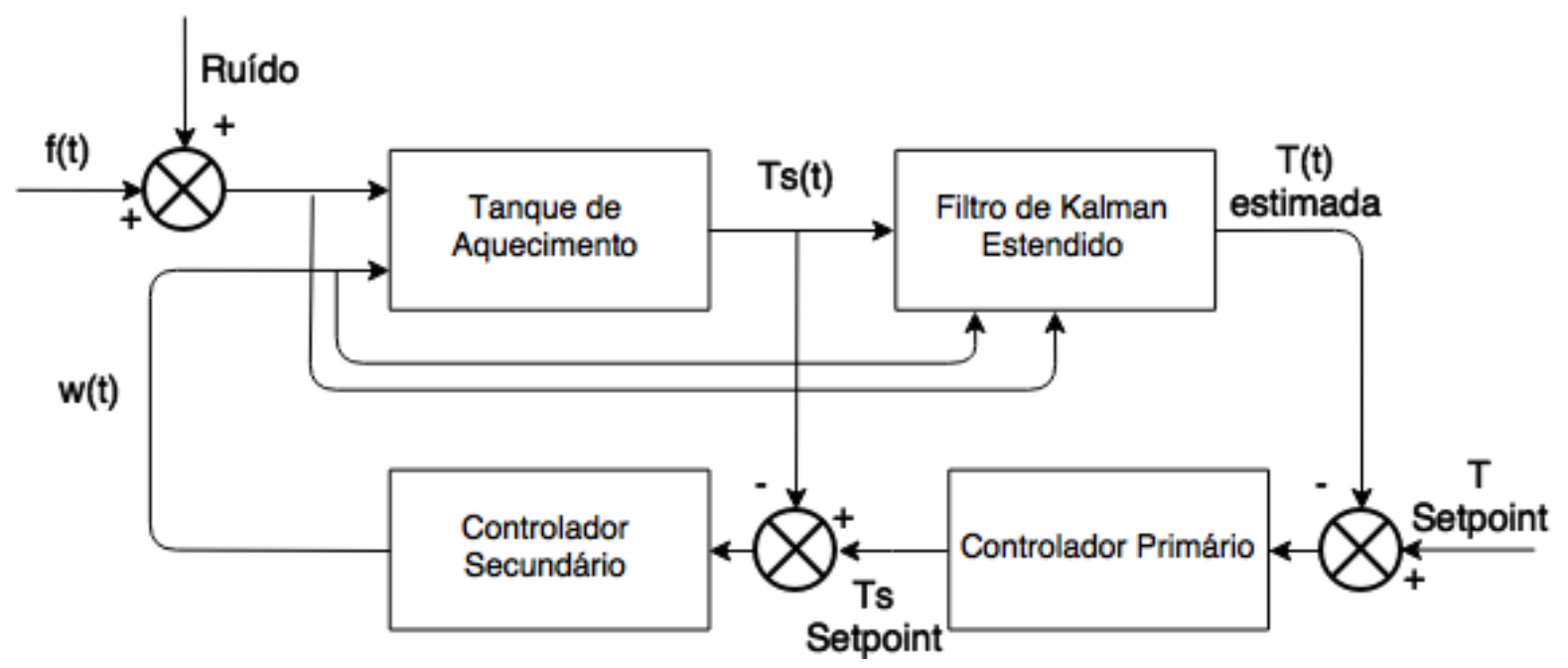

Fonte: o autor.

\section{FILTRO DE KALMAN ESTENDIDO}

O Filtro de Kalman (FK) é um procedimento matemático iterativo, que utiliza um conjunto de equações e dados de entrada para estimar e predizer o valor real de variáveis com erros de medição, ou atua como observador de estados empregando medições secundárias para estimar o valor de variáveis de difícil medição (GREWAL e ANDREWS, 2001). O Filtro de Kalman Estendido (FKE) é a versão não-linear do FK convencional linear. Dado um modelo composto por variáveis de entrada (u), de estado (x) e de saída (y), o algoritmo de atuação do FKE é formado pelas equações de 3 a 7, abaixo.

$$
\hat{x}_{k \mid k-1}=f\left(\hat{x}_{k-1 \mid k-1}, u_{k-1}\right)
$$




$$
\begin{aligned}
& P_{k \mid k-1}=A_{k-1} P_{k-1 \mid k-1} A_{k-1}^{T}+Q_{k} \\
& K_{k}=P_{k \mid k-1} C_{k}^{T}\left(C P_{k \mid k-1} C_{k}^{T}+R\right)^{-1} \\
& \hat{x}_{k \mid k}=\hat{x}_{k \mid k-1}+K_{k}\left[y_{k}-h\left(\hat{x}_{k \mid k-1}\right)\right] \\
& P_{k \mid k}=\left(I-K_{k} C_{k}\right) P_{k \mid k-1}
\end{aligned}
$$

Onde $A_{k}$ e $C_{k}$ são, respectivamente, as matrizes jacobianas das variáveis de estado e de saída. Para o processo em estudo, as variáveis de entrada são: a vazão de vapor (w) e de alimentação do tanque (f); as variáveis de estado são a temperatura do tanque (T) e a temperatura da serpentina ( $\mathrm{T}_{\mathrm{S}}$ ); e a variável de saída (medição) é a temperatura da serpentina. Foi inserido um ruído gaussiano de média zero e covariância igual a 0,1 , que se adciona à $f$ do tanque, e considerado um ruído de medição com covariância igual a 0,0001.

\section{RESULTADOS E DISCUSSÕES}

Inicialmente os resultados obtidos dizem respeito ao comportamento do processo em malha aberta, quando foi inserido uma perturbação, $-10 \%$ no tempo igual a 100 min, na vazão de alimentação do tanque, gráfico da Figura 3.a). Pode-se observar no gráfico da Figura 3.b), que os valores de temperatura estimados utilizando o FKE aproximam-se dos valores reais da temperatura obtidos pela simulação.

Figura 3 - Comportamento das variáveis do processo em malha aberta.

a)

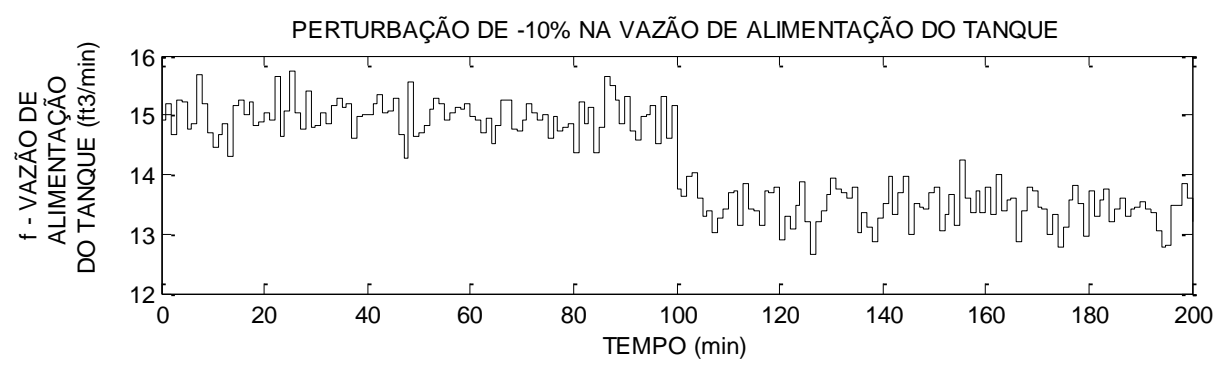

b)

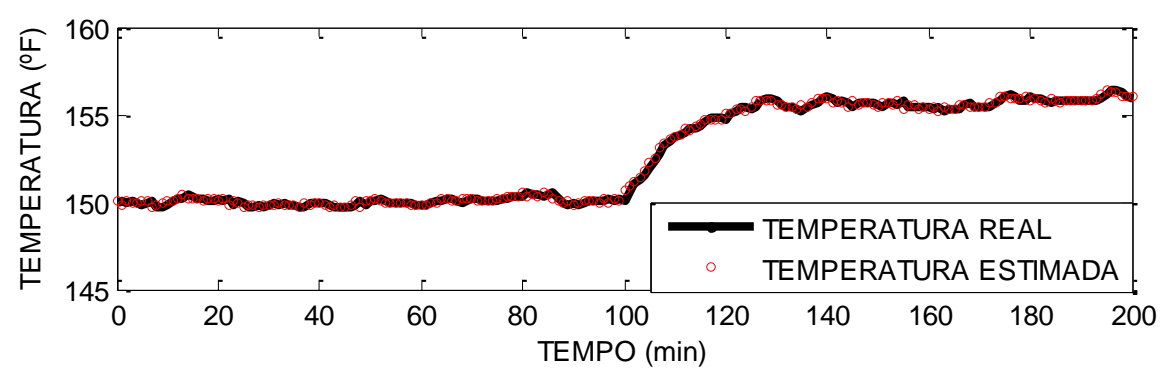


A estimativa foi avaliada utilizando os critérios de Média Quadrática do Erro (MSE) e Raiz da Média Quadrática do Erro (RMSE), que forneceram, respectivamente, os valores de 0,1249 e 0,1590 . Esses valores correspondem a um erro de estimativa de aproximadamente $0,106 \%$.

Foi implementado um controlador proporcional-integrativo (PI) utilizando os valores estimados pelo FKE como medição a fim de manter a variável de processo (temperatura do líquido no interior do tanque) no valor desejado (setpoint, SP) de $150^{\circ} \mathrm{F}$. Pode-se observar o comportamento da variável de processo, PV, no gráfico da Figura 4. No tempo de 100 min, quando ocorre a perturbação na vazão de alimentação do tanque, a PV apresenta uma sobreelevação percentual de aproximadamente $2 \%$.

Figura 4 - Comportamento da variável do processo com controle inferencial realimentação.

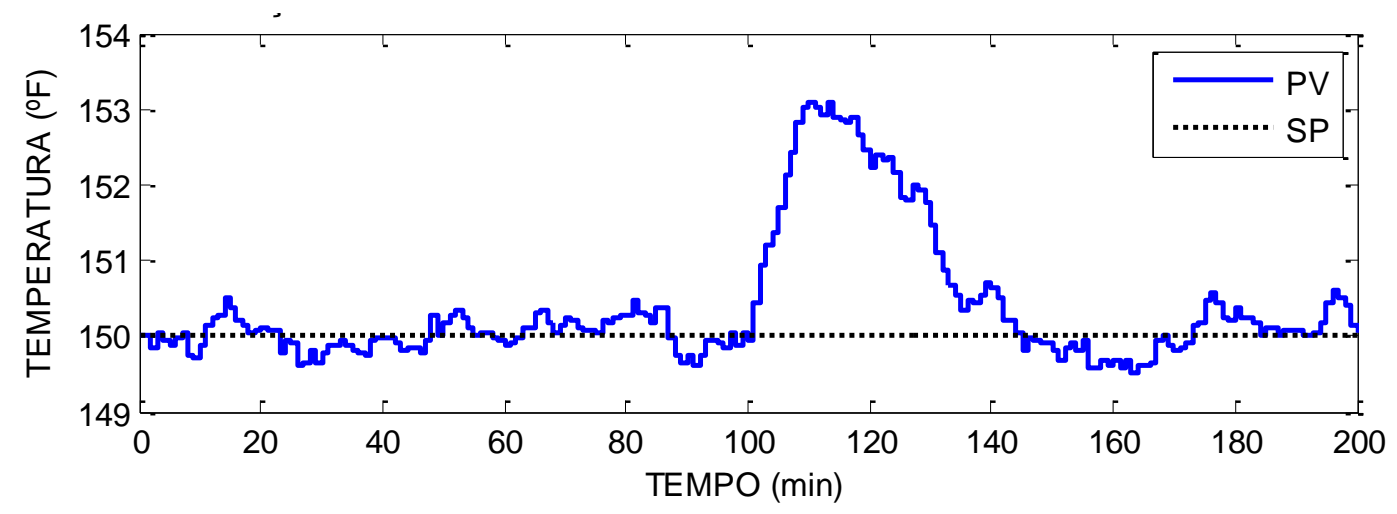

Em seguida, foi implementada a estratégia de controle cascata. Esta estratégia consiste na adição de um controlador secundário com o objetivo de manter a temperatura da serpentina no valor desejado. Este valor desejado consiste na saída do controlador primário que atuava anteriormente no controle convencional realimentação. A Figura 5 apresenta 0 comportamento da PV (temperatura do líquido no tanque) frente à mesma perturbação dos casos anteriores.

Figura 5 - Comportamento das variáveis do processo com controle inferencial cascata.

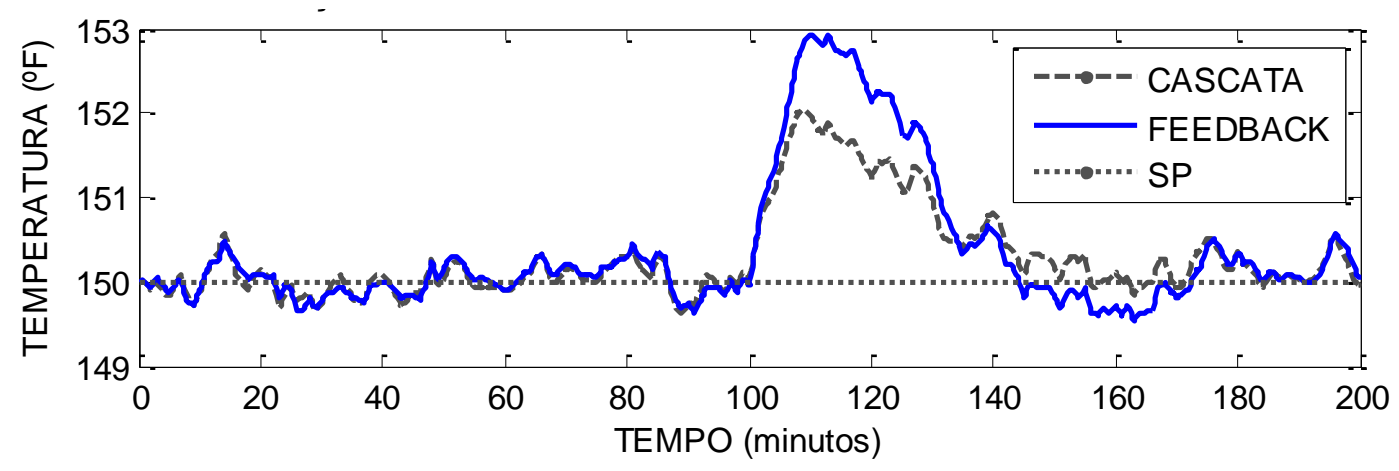

A comparação entre o erro em malha aberta, com controle realimentação convencional e com controle cascata está apresentada na Tabela 2, que contém os critérios de Integral do Erro Absoluto (IAE), Integral do Erro Absoluto vezes o Tempo (ITAE), Integral do Erro 
Quadrático (ISE) e Integral do Erro Quadrático vezes o Tempo (ISAE) para cada caso, em um tempo de simulação igual a 200 minutos.

Tabela 2 - Critérios de integral do erro

\begin{tabular}{|c|c|c|c|c|}
\hline Caso & IAE & ISE & ITAE $\left(\times 10^{4}\right)$ & ISAE $\left(\times 10^{4}\right)$ \\
\hline \hline Malha aberta & 535 & 2830 & 8,099 & 44,38 \\
\hline Realimentação & 103 & 176,7 & 1,179 & 2,058 \\
\hline Cascata & 73,34 & 75,14 & 0,8268 & 0,8687 \\
\hline
\end{tabular}

\section{CONCLUSÕES}

Observou-se que a estimativa fornecida pelo FKE consiste de valores com erro de menos de $0,106 \%$ do valor real. Essa estimativa foi então utilizada na implementação de duas estratégias de controle, a convencional realimentação e o controle cascata. Percebeu-se que, em relação à malha aberta, ambas as estratégias de controle forneceram melhores valores dos critérios de erro integrais e conseguiram manter as variáveis no valor desejado, podendo-se concluir que a filtragem do sinal foi imprescindível no sucesso da etapa de sintonia do controlador. De fato,, o controle cascata apresentou melhor desempenho do que o controle convencional realimentação, podendo-se concluir que a estimativa efetuada pelo FKE pode ser utilizada em estratégias de controle avançado. Por fim, a metodologia desenvolvida para o implemento do FKE, portanto, mostrou-se satisfatória para estimar com boa precisão variáveis de estado de processo.

\section{REFERÊNCIAS}

AGUIRRE, L. A. Introdução à identificação de sistemas: Técnicas lineares e não-lineares aplicadas a sistemas reais. $2^{\mathrm{a}}$ revista e ampliada. Belo Horizonte: Editora UFMG, 2004. 728 p.

GREWAL, M. S.; ANDREWS, A. P. Kalman filtering: theory and practice using MATLAB, 2001.

MORAIS Jr., A. A. Elaboração de um analisador virtual utilizando sistema híbrido neurofuzzy para inferenciar a composição em um processo de destilação. $82 \mathrm{f}$. Dissertação (Mestrado em Engenharia Química). Universidade Federal de Alagoas, Maceió. 2011.

SMITH, C. A. E CORRIPIO, A. Princípios e prática de controle automático de processo. $3^{\mathrm{a}}$ Edição, Editora Gen e LTC, 2008. 